\title{
Contributio ad Floram Asiaticam Novam-Guineam inclusem
}

\author{
acutore Tetsuo Koyama* \\ 小山鉄夫：アジアの植物への笴与
}

Acceptum Martio 10, 1956

Carex hypolytrifolia T. Koyama, spec. nova ad sectionem Mapaniifoliae; omnibus speciebus in hac sectione huc usque descriptas habitu multo minore, foliis brevioribus angustioribusque flaccidis tenuibus, paniculis partialibus laxioribus ita rachi visibili valde dissimilis est. (Fig. 1)

Perennis haud caespitans, rhizomate crassiusculo lignoso collo ascendente squamis fuscis obtecto, radicibus validis. Folia omnia radicaria ex unico rhizomate circa 7 fascicularia late linearia usque linearioblanceolata $28-52 \mathrm{~cm}$ longa medio $15-20 \mathrm{~mm}$ lata laete viridia flaccida plana praeter margines minute scabres laevia apice

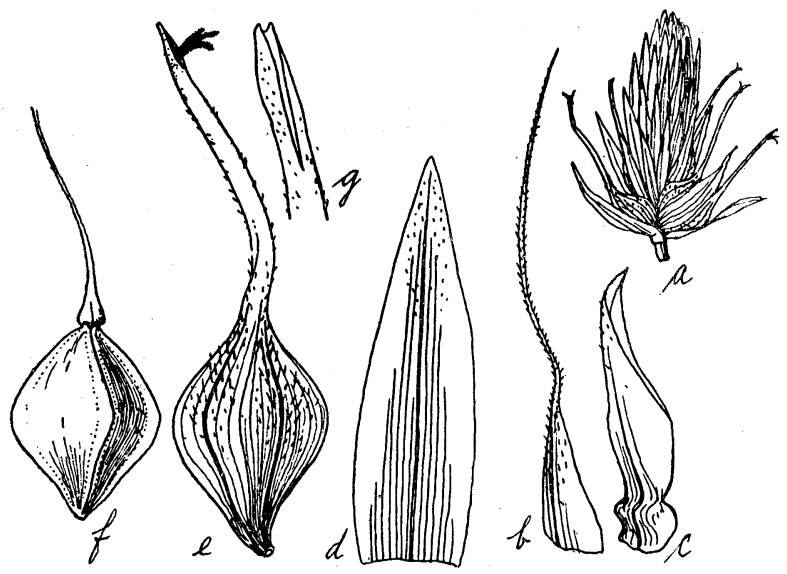

Fig. 1. Carex hypolytrifolia, n. sp. a. spicula; b. bracteola spiculae; c. prophyllum basis spiculae; d. squama floris foeminei ; e. utriculus; f. nux cum stylo; g. os rostri utriculi.

subsensim breviuscule acuta basi in vaginas breves antice fulvohyalinas dorso purpurascentinervatas gradatim attenuantia. Vaginae basilares aphyllae vaginiformes membranaceae fuscae non fibrososolutae. Culmus centralis e fasciculo foliorum solitarius erectus 4-5 dm altus graciles 1-1.5 mm crassus obtuse trigonus laevissimus. Inflorescentia erecta paniculata decomposita subcontigua oblonga $15 \mathrm{~cm}$ longa $4 \mathrm{~cm}$ lata; paniculae partiales 3 singulae ovoideae erectae $3-6 \mathrm{~cm}$ longae $2.5-4 \mathrm{~cm}$ latae densiusculae 3-5-spicatae longe exsertopedunculatae, pedunculis acute triquetris ad

* Botanical Institute, Faculty of Science, University of Tokyo. 東京大学理学部植物学教窒 
angulo hirtoscabris, rachi acute triquetra scabrangula; bracteae vaginantes inferior subfoliacea, laminâ ad $8 \mathrm{~cm}$ longâ $6 \mathrm{~cm}$ latâ paniculam secundariam suam superante, vaginâ ad $2.5 \mathrm{~cm}$ longâ, superior subspathacea paniculâ suâ multo previor. Spicae ellipsoideae pluri-(3-7-)spiculosae patentes subsessiles, rachi e prophyllo ochreiforme tenuimembranaceo enatâ hispidangulâ, bracteolis squamiformibus dorso breviter hispidis apice scabroaristatis. Spiculae androgynae rectangulariter divergentes ovoideae 8-10 mm longae maturitate 5-7 mm crassae dense multiflorae, parte foemineâ 3-6-florâ eâ masculâ ellipsoideâ multiflorâ breviore; prophylla spathaceoutriculiformia membranacea fulva superne sparsim hispiclula, bracteolis eae spicarum similibus. Squamae foemineae lanceolatae vel oblongolanceolatae $3.8-4.5 \mathrm{~mm}$ longae 0.8-1.2 mm latae membranaceae inferne glabrae superne minute hirsutae dilute fulvae a basi ad apicem acutiusculam hyalinam utrinque sensim attenuantes dorso subaequliter pluri-(ad 17-) nerviae. Utriculi maturitate patentes squamam superantes 6-7 mm longi $1.5 \mathrm{~mm}$ lati membranacei olivaceovirentes praeter costas 2 tenuiter multinervosi a medio ad apicem hispidi, e parte inferiore rhomboideo-ovale vere trigonâ basi subito cuneatocontractâ subestipitatâ apice etiam subito attenuatâ in rostrum gracile perlongum $3-3.5 \mathrm{~mm}$ longum $0.2 \mathrm{~mm}$ latum primo erectum demum plerumque flxuosum ad angulos sparasim spinulosohispidum transeuntes, ore hyalino scabrido profunde oblique fisso. $N u x$ arcte inclusa late rhomboidea 2-2.2 $\mathrm{mm}$ longa $1.5 \mathrm{~mm}$ lata vere triquetra facie concaviuscula atrofusca ad angulos prominentes flavida apice basique abrupte cuneatocontracta; stylus longus gracilis flexuosus basi breviter conicoincrassatus, stigmatibus 3 recurvis brevibus papulosis.

A N N A M: Bun Mo, 800m. alt Leg. B. Hayata, sin. num.! -holotypus in TI.

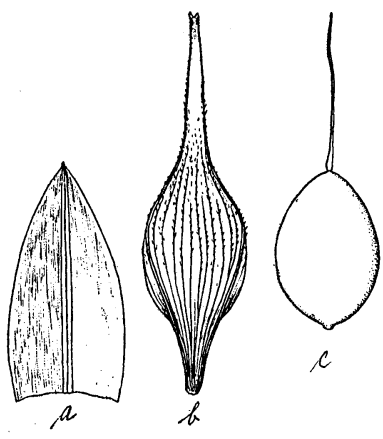

Fig. 2. Carex megacarpa,

n. sp. a, squama floris foeminei; b. utriculus; c. nux. (Kostermans, 6231)

Carex megacarpa T. Koyama, spec. nova in sectione Graciles; proxime ad hanc speciem accedit C. nachiana Ohwi ex Japonia descripta, tamen a planta javanensi utriculis late ovalibus $3.5-4 \mathrm{~mm}$ longis, habitu elatiore etc. satis distinguitur, et $\boldsymbol{C}$. autumnalis Ohwi, etiam planta Japoniae australis, est valde distincta ab hac spiculis sexu distinctis, utriculis vix $3 \mathrm{~mm}$ longis._-? C. brunnea Thunb. var. dolichocarpa Nelmes in Kew Bull. 1950: 201 (1950), e descriptione. (Fig. 2)

Perennis, rhizomate abbreviato lignoso perdense caespitoso, radicibus validis. Culmi erecti 60-100

$\mathrm{cm}$ alti vere triquetri sursum scabridi nutantes inferne laeviusculi remote 1-3.foliati basi vaginis obtecti. Vaginae basilares aphyllae vel laminatae fuscobrunneae dorso demum in fibras sparse solutae antice membranaceae dein in fibras reticulatim fissae. Folia radicalia culmorumque linearia 1/2-2/3-culmo aequilonga 2-3 $\mathrm{mm}$ lata plana vel subconduplicata scabrida apice longissime acuminata basi 
in vaginas longas (5-7 cm in foliis culmorum) antice tenuimembranaceas fulvas reticulatim nervulosas vix attenuata. Spiculae 8-13 superiores singulae geminaeve inferiores in paniculis secundariis perlaxis dispositae paniculam gracilem formantes, omnes androgynae oblongo- vel vere cylindricae $1.5-3 \mathrm{~cm}$ longae maturitate $3-4 \mathrm{~mm}$ crassae densiuscule pluriflorae, parte foeminea ea mascula 2-4-plo superante, pedunculo gracili scabro longe exserto. Bracteae inferiores subfoliaceae panicula partiali sua paullo longiores basi longe vaginantes, caeterae bracteolaeque spathaceae margine anguste fulvomembranaceae apice interdum aristatae. Squamae foemineae ovatoellipticae vel ellipticae $3-3.5 \mathrm{~mm}$ longae ca. $1.5 \mathrm{~mm}$ latae tenuiter membranaceae medio minute fuscolineolatae latere fuscotinctae margine peranguste hyalinae apice acutiusculae obtusiusculaeve, carina late virente uninervia. Utriculi squama longiore latioreque suberecti ovato-oblongi vel oblongeoelliptici $4-5.5$ (-6) $\mathrm{mm}$ longi medio 1.3-1.5 mm lati planoconvexi vel biconvexi membranacei distincte plurinervosi maturitate fulvi, praecipue supra medium marginibusque versus apicem hispiduli, deorsum subsensim contracti in stipitem glabrum $1 / 2-2 / 3 \mathrm{~mm}$ longam, sursum sensim contracti in rostrum rectum 1.5-2 $\mathrm{mm}$ longum antrorsim hispiduloscabrum, ore hyalino oblique fisso. $N u x$ arcte inclusa ovalis $2.5-3 \mathrm{~mm}$ longa $1.4-1.7 \mathrm{~mm}$ lata biconvexa flavens apice basique rotunda mucronulata, stylo longo recto basi subaequali, stigmatibus 2 rostro paullo longioribus.

J A V A: in sylva montis, Lawn super Tjimcrosewu, Sarangan. Leg. J. H. Kern, 8672 ! - holotypus in TNS. Sine loco speciali. Leg. Kostermans, 6231 !.

\section{Carex (Ischnostachyae) ischnostachya Steudel}

var. fastigiata $\mathrm{T}$. Koyama, var. nova, a typo diversa utriculis non vel brevissime rostratis 2.8-3.5 mm longis, spiculis superioribus 3-5 apice culmi fastigiatis. $\boldsymbol{C}$. subtumida Ohwi ab hac recedit habitu robustiore, culmo valde scabro, rhizomate longe stolonifero etc.

$\mathrm{R}$ Y U K Y U: $\mathrm{m}$. Tanimadake, Hajimura in ins. Okinawa. Leg. T. Amano, 7586 ! - hyiotypus in TI.

Carex (Stellulatae) omiana Franchet et Savatier

var. perileia (S. T. Blake) T. Koyama, stat. nov.-C. perileia S. T. Blake in Journ. Arn. Arbor. 28: 102 (1947); Nelmes in Reinwardtia 1: 441 (1951). Distrib. in Nova-Guinea endemica.

Eriocaulon Omuranum T. Koyama, spec. nova e sectione Spathopeplus ; E. Takae Koidz. et $E$. hondoensi Satake proximum videtur, tamen ab hoc bracteis involucri apice acutissimis non dentatis floribus duplo superantibus medio latiuscule viridibus et ab illo habitu multo minore, petalis intus glaberrimis recedit. (Fig. 3)

Annuum gracile acaulescens, radicibus fibrosis albis transverse septatis. Folia pauca linearia $1 / 2-1 \mathrm{~mm}$ lata $5.5-12 \mathrm{~cm}$ longa flaccida circiter 4-nervata sep- 
tatonodulosa versus apicem longe acuminatam gradatim attenuantia. Pedunculi 1-4 ex unica planta erecti capillares $10-15 \mathrm{~cm}$ alti vix torti 4 -costati, costis anguste membranaceis. Vaginae basilares basem pedunculi perlaxe circumdantes cylindricae 1.5-4.5 cm alti 1-1.7 $\mathrm{mm}$ lati septatonodulosae apice hyalinae oblique fissae. Capitulum turbinatum $4.5-5.5 \mathrm{~mm}$ altum $4-6 \mathrm{~mm}$ in diametro omnino glaberrimum 9-17-florum; bracteae involucrantes (4-) 5-6 erectopatentes lanceolatae vel anguste lanceolatae 2. 5-6 $\mathrm{mm}$ longae $0.8-1.2 \mathrm{~mm}$ latae sursum gradatim attenuatae apice longe subulatocuspidatae medio late virides membranaceae marginibus utrinque latiuscule albohyalinae; receptacrum glabrum. Flores foeminei marginales cum stipite circiter $1 / 2 \mathrm{~mm}$ longo $3.7 \mathrm{~mm}$ longi; bractea lanceolata oblongolanceolatave hyalina pallida $2.5 \mathrm{~mm}$ longa apice acutiuscula; calyx ovalis $2.3 \mathrm{~mm}$ longus hyalinus pallidus obsoletissime trinervulosus ex toto glaberrimus apice contractus tridentatus, dentibus deltoideis centrale quam laterales 2 breviore angustioreque omnibus glabris; petala 3 libera lanceolata vel ovatolanceolata alba $3.5 \mathrm{~mm}$ longa calycem paullo excedentia basi subabrupte contracta stipitata intus extraque glabra apice acutiuscula mucronata glandula unica coronata; ovarium triloculare, stylo longo recto, stigmatibus 3. Flores masculi centrales $1.7 \mathrm{~mm}$ longi; bractea oblanceolata hyalina pallida flore paullo brevior apice rotunda; calyx spathiformis hyalinus pallidus apice obtusus vel leviter emarginatus omnino glaber; corolla subteres apice trilobata, lobis aequalibus oblongis obtusis; stamina 6 lobis corollae longiora, antherae globosae nigrae.

J A P O N I A : circa lacum Shirakabako, Honshu. Leg. Toshiro Omura, 6 aug., 1951 !holotypus in TNS.

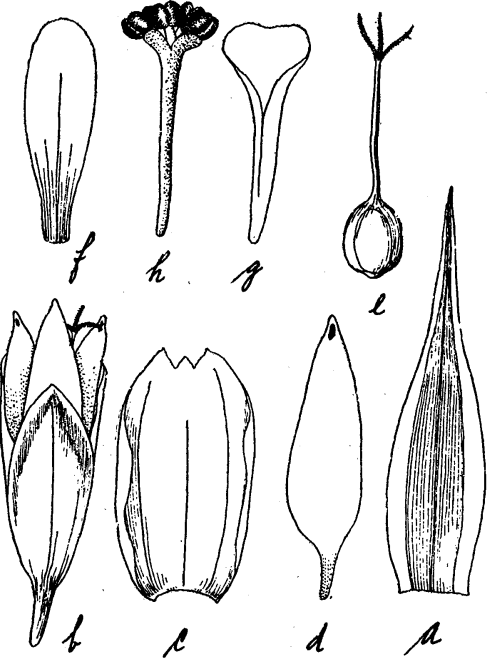

Fig. 3. Eriocaulon Omuranum, n. sp. a. bractea involucri; b. flos foemineus cum bractea sua; c. ejusdem calyx ; d. ejusdem petalum; e. pistilum ; f. bractea floris masculi; g. calyx floris masculi; h. corolla floris masculi cum staminibus. Omnes e typo delineabantur.

Scirpus Ohwianus T. Koyama, spec. nova e vicinia Scirpi juncoidei Roxb. a qua nuce obovata non orbiculare, spiculis pluribus densius floriferis culmis robustioribus ample distinctus-'S. juncoides (non Roxb.)': Ohwi in Mem. Coll. Sci. Kyoto Imper. Univers. ser. B, 18 (1):113 (1943), pro parte, excl. var. Hotarui Ohwi. (Fig. 4)

Herba annua caespitans fere erhizomata, radicibus fibrosis multis fuscescentibus. Culmi erecti 3-6 dm alti 1.2-4 (raro ad 5) $\mathrm{mm}$ crassi tereti obscure pluricostulati laeves laete virides opaci basi vaginis paucis obsiti. Folia omnia in vagias aphyllas reducta. Vaginae basilares inferiores tenuiter membranaceae fuscae squamiformes usque subspathaceae apice profunde oblique fissae obtusae vel plus minus emar- 
ginatetae, superiores cylindricae, summa ad $13 \mathrm{~cm}$ longa dorso viridis antice hyalina ore oblique secta mucronata. Inflorescentia pseudolateralis capitatocontracta (1-) 2-9 (-12)-spiculosa. Bractea unica teres culmum continuans erecta $5-15 \mathrm{~cm}$ longa inferne antice unisulcata. Spiculae oblongocylindricae radiantes 9-18 $\mathrm{mm}$ longae $3.5-5 \mathrm{~mm}$ in diametro dense pluriflorae fulvovirentes. Squamae imbricatim dispositae spissae ovales $3.5-4 \mathrm{~mm}$ longae $1.8-2.7 \mathrm{~mm}$ latae valde naviculares membranaceae latere utrinque fulvotinctae apice rotundatae otusae vel emarginatae mucronatae margine latiuscule albohyalinae inferne aequaliter tenuinerviae, costa late viridi 1-3-nervia. Nux obovata subbiconvexa $1.8-2 \mathrm{~mm}$ longa circiter $1.5 \mathrm{~mm}$ lata basi abrupte attenuata obdeltoidea apice rotundocontracta mucronata facie

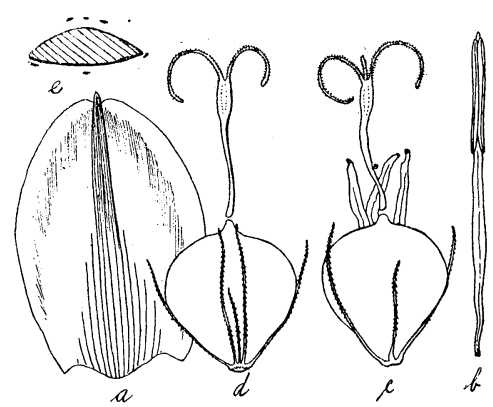

Fig. 4. Scirpus Ohwianus, n. sp. a. squama; b. stamen; c. nux cum setis, filamentis et stylo, postice visa; d. eadem cum setis styloque a dorso visa; e. sectio transversalis nucis (e typo). nitida plus minus zonatorugulosa maturitate nigricans. Setae hypogynae 4-6 tenues fluvescentes praeter partem basilarem retrorsim subdense spinulososcabrae, 4 nucem aequantes paullo superantesve, ceterae cum 1/2-2/3-nuce aequilongae. Stylus rectus $2-2.2 \mathrm{~mm}$ longus basi aequalis sub stigmatibus subcompressus, stigmatibus 2 sed saepe cum rudimento brevi unico ita ut videtur trifidis. Stamina 3 , filamentis planis, antheris $1.3 \mathrm{~mm}$ longis, connectivo deltoideo breve.

J A P O N I A circa Yatsumi, Prov. Kadzusa. Leg. T. Koyama, 14591!-holotypus in TNS. Distrib. sp. Japonia (Honshu, Kyushu), Ryukyus.

Nota: Haec species cum Scirpo Hotarui Ohwi saepissime confusa est, tamen ab illo funditus diversa est:

A. Culmus crassior obsolete pluricostulatus; spiculae 3-7; oblongae breviter cylindricaeve; nux biconvexa, styli ramis 2 .

S. Ohwianus, n. sp.

AA. Culmus gracilior laevissimus non costulatus nec angulatus in vivo plus minus nitidulus; spiculae 1-3 ovoideae globoso-ovoideaeve; nux adpresse triquetra, facie postica concaviuscula, styli ramis semper 3 .

S. Hotarui Ohwi

Poa finitima T. Koyama, spec. nova ad seriem Pratenses, a Poa shinanoana Ohwi lemmate antherisque brevioribus et habitu minore praecipue distat. (Fig. 5)

Gramen perenne, stolonibus gracilibus perlongis horizontaliter repentibus fibris fuscis sparse obtectis. Culmus ex unico rhizomate solitarius erectus $45-55 \mathrm{~cm}$ altus 2-3-nodosus basi geniculatus circ. $1 \mathrm{~mm}$ crassus vaginis fuscis fulvisve obsitus, remote pauci-(saepissime 2)-foliatus laevis plus minus nitidulus. Folia linearia, laminis glabris vagina brevioribus $2-2.5 \mathrm{~mm}$ latis usque ad $6 \mathrm{~cm}$ longis, vaginis infra medium antice connatis 5-12 cm longis internodio brevioribus, ligulis hyalinis rotundis fere $1 \mathrm{~mm}$ longis. Panicula oblongo-ovata vel oblonga densiuscula sub- 
erecta 6-12 cm longa $3.5 \mathrm{~cm}$ lata, ramis (2-) 3-5-nis capillaribus flexuosis scabriusculis post anthesi patulis 3-5-spiculatis basi nudis. Spiculae compressae 3-4-florae cine.

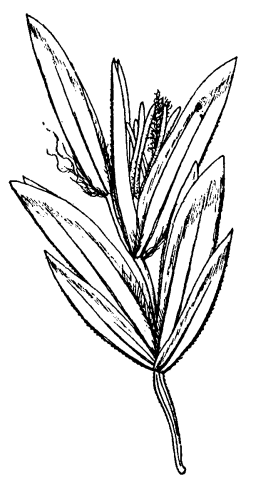

Fig. 5. Poa finitima n.sp. Spicula (e typo). reovirentes vel purpurascenticinereae 5.5-7 mm longae; glumae steriles lanceolate acuminate $3-3.5: 3.7-4 \mathrm{~mm}$ longae trinervatae, nervis 2/3-3/4-glumae attingentibus, carina sursum minute scabra; lemmata anguste oblonga vel lanceolato-oblonga 4-4.5 $\mathrm{mm}$ longa apice latiuscule hyalina acutiuscula praeter carinam minute scabram latere utrinque 2-nervosa basi cuneatoangustata lana longa cincta, carina ad $1 / 2$ nervis marginalibus ad $1 / 3$ proprius usque pilosis, nervo intermedio nudo; paleae lemmate paullo breviores $3.5-4 \mathrm{~mm}$ longae hyalinae apice rotundotruncatae; antherae lineares $1.5-1.7 \mathrm{~mm}$ longae.

J A P O N I A : in resione alpina montis Kiso-Komagatake, prov. Shinano. Leg. K. Machida, s. n. !-holotypus in TI.

Uncinia Ohwiana T. Koyama, spec. nova distinctissima ex affinitate $U$. caespitosae quae spiculis multo longioribus perlaxe florentibus valde dissimilis est. Sectio Stenandrae. (Fig. 6)

Perennis laxe caespitans, rhizomate breviuscule repente estolonifero. Culmi erecti $4-5.5 \mathrm{~cm}$ alti graciles rigidi acute triquetri striati supra medium scabri. Folia anguste linearia rigida $1.5-2 \mathrm{~mm}$ lata culmum aequantia vel paullo superantia plicata canaliculata margine scaberrima apice sensim attenuata longe acuminata. Vaginae basilares fuscae fuscorubentesve rigidae aphyllae inferiores subsquamiformes. Spicula solitaria anguste linearia 5-7 $\mathrm{cm}$ longa $2(-3) \mathrm{mm}$ lata perlaxe florens apice in partem masculam 10-15 (-20) $\mathrm{mm}$ longam vix attenuata, rhachi saltem infra medium visibili. Bractea nulla. Squama foeminea ovato-oblonga 5-6 $\mathrm{mm}$ longa rigida brunnea fuscave partim castanea apice acultiuscula basi fere angustata utriculum amplectens, costa latiuscula 1-3-nervosa resinosolineolata. Utriculus erectus linearioblongus vel lanceolatus squamam paullo superans 6-6.5 (-7) $\mathrm{mm}$ longus adpresse trigonus glaberrimus maturitate fuscatus tenuiter plurinervulosus basi sensim attenuatus in stipitem longiusculum apice subsensim attenuatus in rostrum breviusculum conicocylindricum, ore oblique secto margine anguste albohyalino. $N u x$ arcte inclusa linearioblonga 3-3.5 $\mathrm{mm}$ longa obtuse trigona apice basique subito contracta, stylo brevi basi paullo incrassato subconico supra medium usque

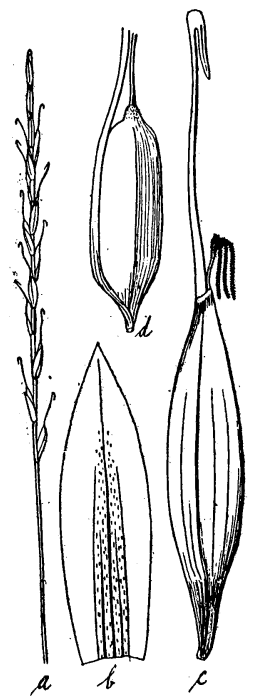

Fig. 6. Uncinia Ohwiana, n. sp. a. spicula; b. squama floris foeminei ; c. utriculus cum racheola et stigmatibus ; d. nux cum parte racheolae (e typo). trifido. Racheola setiformis utriculum duplo superans recta apice hamata. Filamenta filiformia elongata.

N o v A-G U I N E A: sine loco speciali. TNS. no. 92552 !- holotypus. 


\title{
Summary
}

In the present paper, five species and one variety of sedges, grass and pipewort were newly proposed and one sedge was reduced to a varietal rank. A special mention is necessary for a new sedge of Garciles from Java. So far as Thunberg's type, Carex brunnea is characterised by somewhat yellowish-green leaves and very small perigynia 2.5-2.7 $\mathrm{mm}$ long, hence Dr. Kükenthal once considered true $C$. brunnea to be a variety of $C$. gentilis. Although the species of Graciles have very wide deviation, the above plant from Java is not $C$. brunnea. Further, Malaysian plants treated so may possibly be quite different from C. brunnea. The abbreviations TI and TNS are used for the herbaria of the University of Tokyo and of the National Science Museum, Tokyo, respectively.

\section{Physiological Changes in the Germinating Seeds during Low Temperature Treatment II On the Activity of Catalase and Peroxidase}

\author{
by Shôjirô INOUE* and Masaki YAHIRO** \\ 井上昭治郎・八尋正樹：低温处理間の発芽種子の生理的变化 II \\ カタラーゼ及びペルォキシダーゼについて
}

Received December 14, 1955

\section{Introduction}

Many biennial plants, which initiate into the reproductive growth by the long day-length of spring, require chilling of a certain days in the early stage of seedling.

It is considered that the study of the physiological changes in the seedling during the progress of vernalization is a basic matter for dissolving the problem of the reproductive growth.

On the relation between the vernalization and the enzymes, Oveckin and others ${ }^{(1)}$ made researches in the winter wheat, Sapoznikova'2) in Lupinus angustifolius; and B. Sen ${ }^{(3)}$ investigated diastase and phosphatase in the wheat and lipase, catalase and phosphatase in the mustard and diastase in the barley.

In the present experiment the authors dealt with the determination of some enzyme activity, above all, catalase and peroxidase that may have a considerable connection with the respiration.

It is their great pleasure to record here a debt of gratitude to Professor Hitoshi Kojima for his very valuable advice during the work and the authors also wish to express their thanks to Professor Shirakawa for his very helpful suggestions.

* Botanical Laboratory, Faculty of Agriculture, Kyûshû University. 九州大学農学部植物学教室

*k Sericultural Laboratory, Faculty of Agriculture, KagoshimaUniversity. 鹿児島大学農学部養虫 学教室 